\title{
WASP-113b and WASP-114b, two inflated hot Jupiters with contrasting densities
}

S. C. C. Barros ${ }^{1,2}$, D. J. A. Brown ${ }^{3}$, G. Hébrard ${ }^{4,5}$, Y. Gómez Maqueo Chew ${ }^{3,6}$, D. R. Anderson ${ }^{7,21}$, P. Boumis ${ }^{8}$, L. Delrez ${ }^{9}$, K. L. Hay ${ }^{10}$, K. W. F. Lam ${ }^{3}$, J. Llama ${ }^{11}$, M. Lendl ${ }^{12,15}$, J. McCormac ${ }^{3}$, B. Skiff ${ }^{11}$, B Smalley ${ }^{7}$, O. Turner ${ }^{7}$, M. Vanhuysse ${ }^{13}$, D. J. Armstrong ${ }^{3,21}$, I. Boisse ${ }^{2}$, F. Bouchy ${ }^{2}$, A. Collier Cameron ${ }^{10}$, F. Faedi ${ }^{3}$, M. Gillon ${ }^{9}$, C. Hellier ${ }^{7}$, E. Jehin ${ }^{9}$, A. Liakos ${ }^{8}$, J. Meaburn ${ }^{14}$, H. P. Osborn ${ }^{3}$, F. Pepe ${ }^{15}$, I. Plauchu-Frayn ${ }^{16}$, D. Pollacco ${ }^{3}$, D. Queloz ${ }^{15,17}$, J. Rey ${ }^{15}$, J. Spake ${ }^{3}$, D. Ségransan ${ }^{15}$, A. H. M. Triaud ${ }^{18,19,15,20}$, S. Udry ${ }^{15}$, S. R. Walker ${ }^{3}$, C. A. Watson ${ }^{21}$, R. G. West ${ }^{3}$, and P. J. Wheatley ${ }^{3}$

${ }^{1}$ Instituto de Astrofísica e Ciências do Espaço, Universidade do Porto, CAUP, Rua das Estrelas, 4150-762 Porto, Portugal e-mail: susana.barros@astro.up.pt

2 Aix-Marseille Université, CNRS, LAM (Laboratoire d'Astrophysique de Marseille) UMR 7326, 13388 Marseille, France

3 Department of Physics, University of Warwick, Gibbet Hill Road, Coventry, CV4 7AL, UK

${ }^{4}$ Institut d'Astrophysique de Paris, UMR 7095 CNRS, Université Pierre \& Marie Curie, 98 bis boulevard Arago, 75014 Paris, France

5 Observatoire de Haute-Provence, Université d'Aix-Marseille \& CNRS, 04870 Saint-Michel l'Observatoire, France

${ }^{6}$ Instituto de Astronomía, Universidad Nacional Autónoma de México, 04510 Ciudad Universitaria, Mexico

7 Astrophysics Group, Keele University, Staffordshire ST5 5BG, UK

${ }^{8}$ Institute for Astronomy, Astrophysics, Space Applications and Remote Sensing, National Observatory of Athens, 15236 Penteli, Greece

9 Institut d'Astrophysique et de Geophysique, Université de Liège, Allée du 6 Août, 17, Bât. B5C, Liège 1, Belgium

10 SUPA, School of Physics and Astronomy, University of St. Andrews, North Haugh, Fife KY16 9SS, UK

11 Lowell Observatory, 1400 W Mars Hill Rd. Flagstaff, AZ 86001, USA

12 Space Research Institute, Austrian Academy of Sciences, Schmiedlstr. 6, 8042 Graz, Austria

13 OverSky, 47 allée des Palanques, BP 12, 33127 Saint-Jean d'Illac, France

14 Jodrell Bank Centre for Astrophysics, University of Manchester, Oxford Rd., Manchester, M13 9PL, UK

15 Observatoire de Genève, Université de Genève, 51 chemin des Maillettes, 1290 Sauverny, Switzerland

16 Instituto de Astronomía, Universidad Nacional Autónoma de México, 22860 Ensenada, Baja California, Mexico

17 Cavendish Laboratory, J J Thomson Avenue, Cambridge, CB3 OHE, UK

18 Centre for Planetary Sciences, University of Toronto at Scarborough, 1265 Military Trail, Toronto, ON, M1C 1A4, Canada

19 Department of Astronomy \& Astrophysics, University of Toronto, Toronto, ON M5S 3H4, Canada

20 Institute of Astronomy, Madingley Road, Cambridge, CB3 OHA, UK

21 Astrophysics Research Centre, School of Mathematics \& Physics, Queen's University Belfast, University Road, Belfast, BT7 1NN, UK

Received 12 May 2015 / Accepted 4 July 2016

\section{ABSTRACT}

Aims. We present the discovery and characterisation of the exoplanets WASP-113b and WASP-114b by the WASP surveys, SOPHIE and CORALIE.

Methods. The planetary nature of the systems was established by performing follow-up photometric and spectroscopic observations. The follow-up data were combined with the WASP-photometry and analysed with an MCMC code to obtain system parameters.

Results. The host stars WASP-113 and WASP-114 are very similar. They are both early G-type stars with an effective temperature of $\sim 5900 \mathrm{~K},[\mathrm{Fe} / \mathrm{H}] \sim 0.12$, and $\log g \sim 4.1$ dex. However, WASP-113 is older than WASP-114. Although the planetary companions have similar radii, WASP-114b is almost four times heavier than WASP-113b. WASP-113b has a mass of $0.48 M_{\text {Jup }}$ and an orbital period of $\sim 4.5$ days; WASP-114b has a mass of $1.77 M_{\text {Jup }}$ and an orbital period of $\sim 1.5$ days. Both planets have inflated radii, in particular WASP-113 with a radius anomaly of $\Re=0.35$. The high scale height of WASP-113b $(\sim 950 \mathrm{~km})$ makes it a good target for follow-up atmospheric observations.

Key words. planets and satellites: detection - stars: individual: WASP-113 - stars: individual: WASP-114 techniques: radial velocities - techniques: photometric

\section{Introduction}

In the last few years there has been a huge increase in the number of transiting exoplanets known mainly due to the Kepler satellite (Borucki et al. 2010). Currently, 2600 transiting exoplanets are known and there are about one thousand unconfirmed candidates. Transiting planet systems are especially valuable because their geometry enables us to derive accurate planetary properties (Charbonneau et al. 2000; Henry et al. 2000). Time series photometry during transit allows the estimation of the orbital inclination and the relative radii of the host star and planet. These can be combined with radial velocity measurements and 
stellar parameters to derive the absolute planetary mass (e.g. Barros et al. 2011a). Hence, the bulk density of the planet can be estimated with good accuracy, giving us insight into its composition (Guillot 2005; Fortney et al. 2007), thus placing constraints on planetary structure and formation models.

Furthermore, follow-up observations of transiting planets gives further insight into their physical properties. Transmission spectroscopy, which consists of measuring the stellar light filtered through the planet's atmosphere during transit, provides information about exoplanet atmospheres (Charbonneau et al. 2002; Vidal-Madjar et al. 2003). Moreover, observation of secondary eclipses (i.e. occultations) offers the potential for directly measuring planetary emission spectra (e.g. Deming et al. 2005; Charbonneau et al. 2008; Grillmair et al. 2008). However, these follow-up observations are currently feasible only for bright stars.

There are only a few hundred transiting exoplanets around stars brighter than $V=13$. These bright host stars enable follow-up observations to better characterise the systems. Therefore, several second generation surveys - ground-based: NGTS, MASCARA, and SPECULOOS and space-based: CHEOPS (ESA), PLATO (ESA), and TESS (NASA) - are being developed to target bright stars. The new Kepler satellite mission K2 (Howell et al. 2014) is making a significant scientific impact by monitoring a large number of fields and a significant number of bright stars at a photometric precision only slightly inferior to the original Kepler mission (e.g. Vanderburg \& Johnson 2014; Barros et al. 2016).

In this paper, we report the discovery of WASP-113b and WASP-114b by SOPHIE, CORALIE, and the WASP-project (Pollacco et al. 2006), the leading ground-based transit survey which has discovered $\sim 150$ exoplanets around stars brighter than $V=13$ mag. The WASP project consists of two robotic observatories: one at the Observatorio del Roque de los Muchachos, La Palma, Canary Islands, Spain, and the other at the South African Astronomical Observatory of Sutherland, South Africa.

The host stars WASP-113 and WASP-114 are similar early G-type stars. Both planets have radii $\sim 50 \%$ greater than Jupiter's, but WASP-113b is less than half of Jupiter's mass while WASP-114b has almost twice the mass of Jupiter. Hence WASP-114b is 4.2 times more dense than WASP-113b. We start by describing the photometric and spectroscopic observations of both systems in Sect. 2 and present the spectroscopic characterisation of the stars in Sect. 3. In Sect. 4 we describe the system analysis and present the results. We finish by discussing our results in Sect. 5.

\section{Observations}

\subsection{SuperWASP observations}

The SuperWASP-North observatory in La Palma consists of eight cameras each with a Canon 200-mm f/1.8 lens coupled to an Andor e2v $2048 \times 2048$ pixel back-illuminated charge coupled device (CCD; Pollacco et al. 2006). This configuration gives a pixel scale of $13.7^{\prime \prime} /$ pixel, which corresponds to a field of view of $7.8 \times 7.8$ sq. deg per camera.

The field containing WASP-113 $(\alpha=14: 59: 29.49 \delta=$ $+46: 57: 36.4$ ) was observed in the period between 2011 March 30 and 2011 June 30 simultaneously by two out of the eight cameras. A total of 29942 good points were collected for WASP-113.

The field containing WASP-114 $(\alpha=21: 50: 39.74 \delta=$ $+10: 27: 46.9$ ) was observed from July 2006 till November 2011.

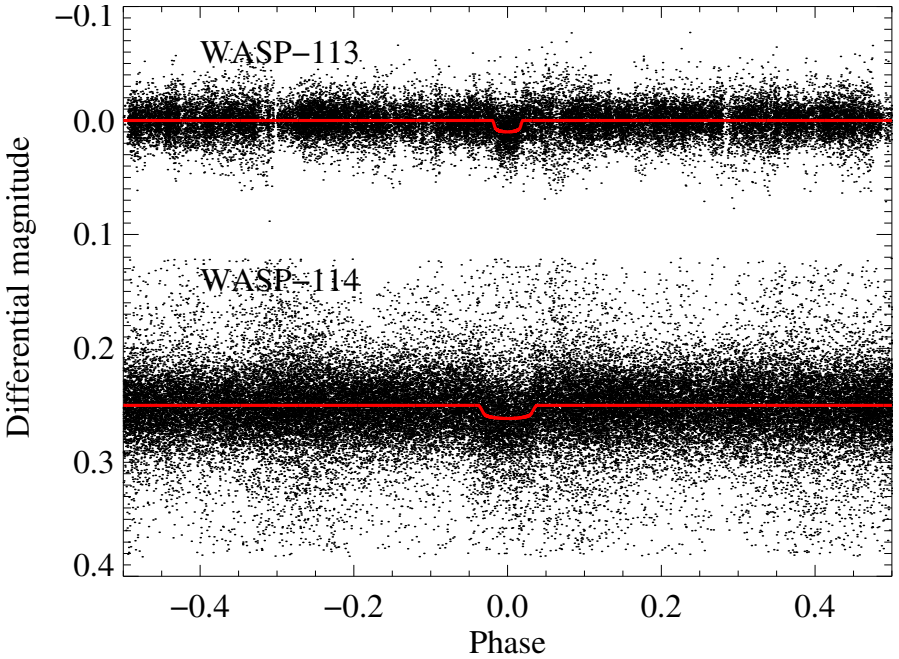

Fig. 1. SuperWASP phase folded light curve for WASP-113 (top) and WASP-114 (bottom). The best-fit transit model described in detail in Sect. 4 is overplotted. The data of WASP-114 was displaced vertically for clarity.

During this period 53673 observations of WASP-114 were collected.

The light curves were analysed with the automatic WASP pipeline. The data are detrended using the algorithms SYSREM (Tamuz et al. 2005) and TFA (Kovács et al. 2005). Subsequently, a transit search is performed which is detailed in Collier Cameron et al. (2006, 2007). Both stars were flagged as transit candidates after passing initial tests for false positives and were recommended for follow-up observations in March 2013 and June 2012 for WASP-113 and WASP-114, respectively. The phase folded WASP light curves of WASP-113 and WASP-114 are shown in Fig. 1.

\subsection{Spectroscopic follow-up}

Radial velocity measurements of WASP-113 were taken with SOPHIE mounted on the $1.93 \mathrm{~m}$ telescope of the Observatoire de Haute Provence (Perruchot et al. 2008; Bouchy et al. 2009). A total of 20 measurements were obtained from the 21 April 2013 to the 2013 of September 16. For WASP-114 both SOPHIE and CORALIE mounted on the $1.2 \mathrm{~m}$ Swiss Euler telescope in La Silla (Baranne et al. 1996; Queloz et al. 2000; Pepe et al. 2002) were employed to obtain radial velocity measurements. Between 2013 July 13 and 2013 October 6, 18 measurements were obtained with SOPHIE and 17 with CORALIE. The data were reduced with the SOPHIE and CORALIE pipelines. The radial velocity errors account for the photon noise.

The radial velocity measurements are given in Table 1 for WASP-113 and in Table 2 for WASP-114. In Fig. 2, we show the phase folded radial velocities for WASP-113b in the top panel and WASP-114b in the bottom panel. The best-fit Keplerian model described in Sect. 4 is superimposed on the data points and we also show the residuals from the model, which show no long term trend.

We performed a bisector span analysis for both stars, which is shown in Fig. 3. For both WASP-113 and WASP114 there are no significant variations of the bisector span with the radial velocities. This supports the planetary nature of the system since in the case of stellar activity or blended eclipsing binaries the bisector can correlate with the radial velocity measurements. 
S. C. C. Barros et al.: WASP-113b and WASP-114b, two inflated hot Jupiters with contrasting densities
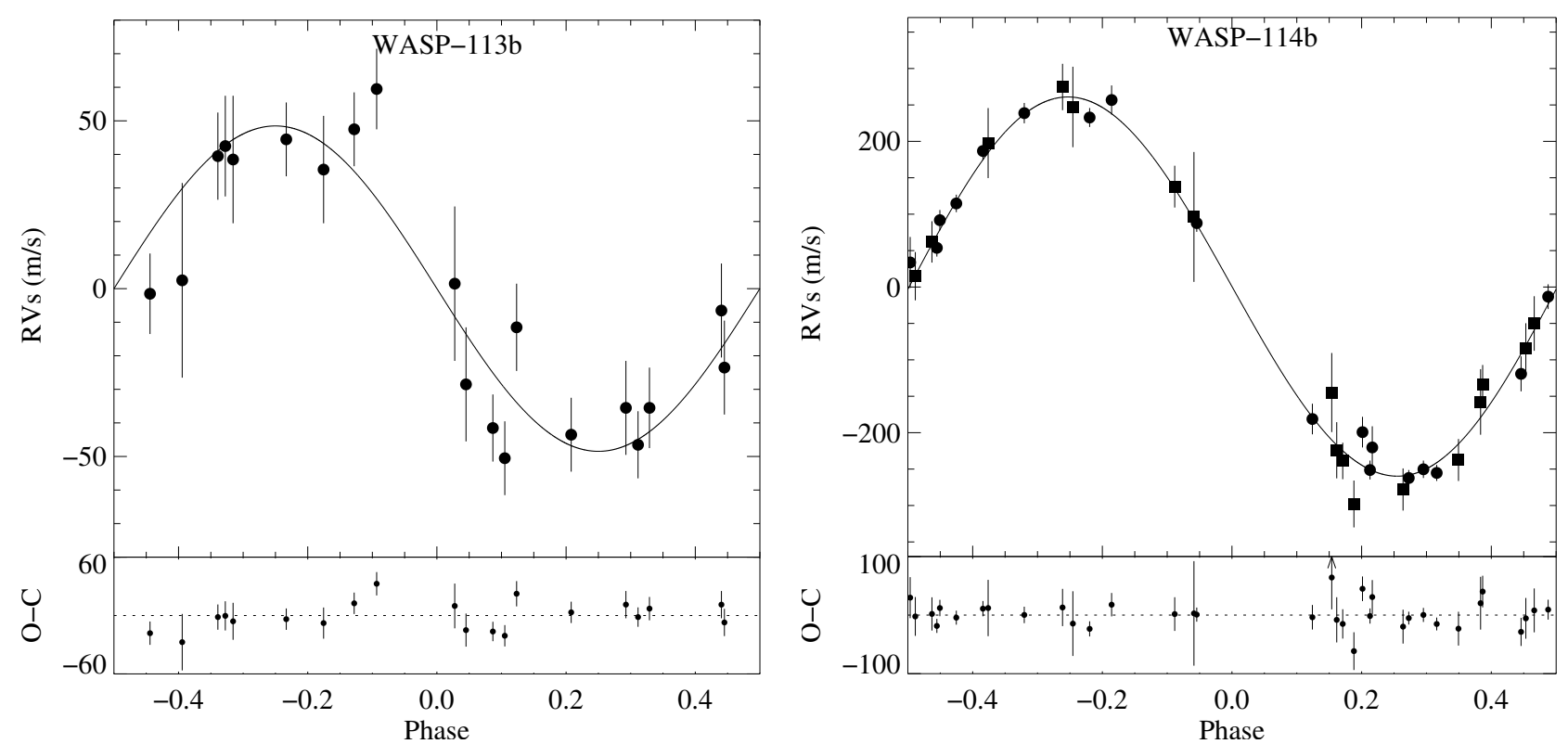

Fig. 2. Phase folded radial velocities of WASP-113 (left) and WASP-114 (right). The observations taken with SOPHIE are plotted as circles and those taken with CORALIE as squares. The overplotted black curve is the most probable fit orbital model and the residuals from these fits are also given (bottom panel). For WASP-113b we impose a circular orbit, while the eccentricity was left free in the model of WASP-114b.
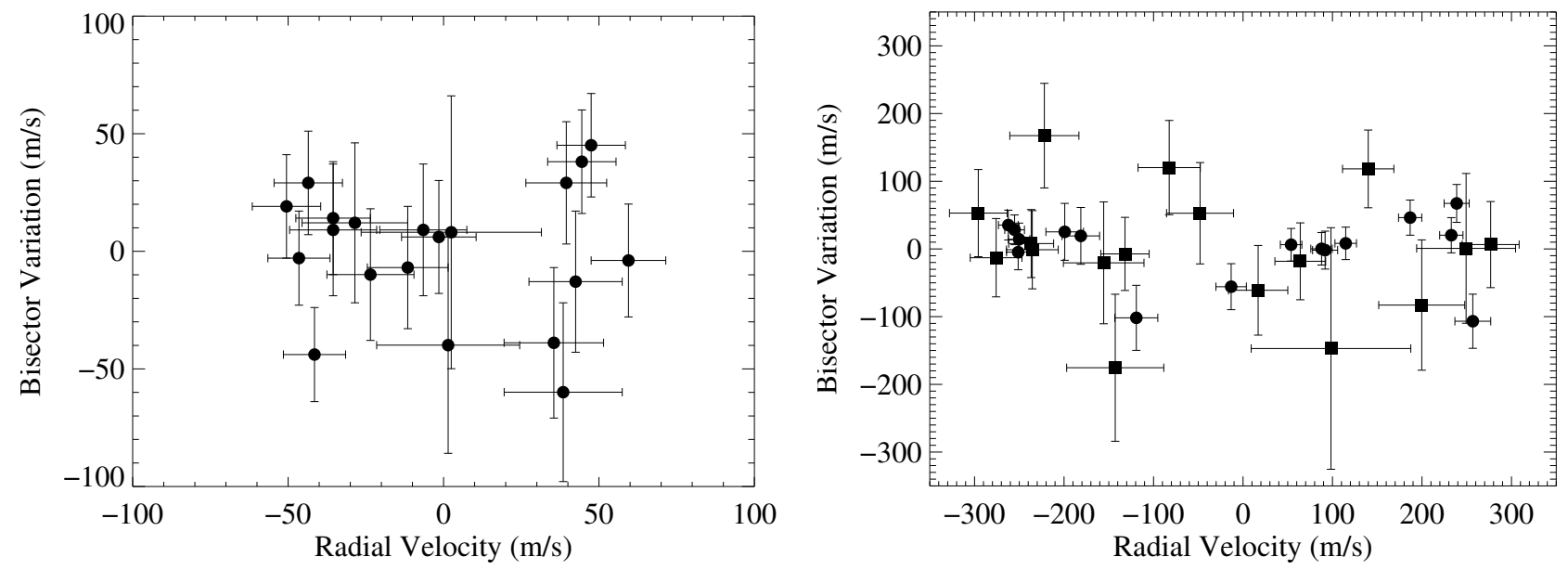

Fig. 3. Bisector span measurements as a function of radial velocity for WASP-113 (left) and WASP-114 (right). SOPHIE data are plotted as circles, while CORALIE are plotted as squares.

\subsection{Photometric follow-up}

To better constrain the transit shape, high precision transit light curves were obtained for both planets. Seven observations were performed for WASP-113 and six for WASP-114; they are summarised in Table 3.

\subsubsection{WASP-113}

Two transits of WASP-113b were observed with the NearInfrared Transiting ExoplanetS (NITES) telescope, the first on 2014 June 06 and the second on 2015 March 10. The NITES telescope is a semi-robotic $0.4 \mathrm{~m}(f / 10)$ Meade LX200GPS Schmidt-Cassegrain telescope installed at the observatorio del Roque de los Muchachos, La Palma. The telescope is mounted with a Finger Lakes Instrumentation Proline 4710 camera, containing a $1024 \times 1024$ pixel deep-depleted CCD made by e $2 \mathrm{v}$. The telescope has a field of view of $11 \times 11$ arcmin squared and a pixel scale of $0.66^{\prime \prime}$ pixel $^{-1}$, and a peak quantum efficiency $>90 \%$ at $800 \mathrm{~nm}$. For more details on the NITES Telescope see McCormac et al. (2014). For these observations, the telescope was defocused slightly to $4.0^{\prime \prime}$ FWHM and 865 images of $20 \mathrm{~s}$ exposure time were obtained with $5 \mathrm{~s}$ dead time between each. Observations were obtained without a filter. The data were bias subtracted and flat-field corrected using PyRAF ${ }^{1}$ and the standard routines in $\mathrm{IRAF}^{2}$, and aperture photometry was performed using DAOPHOT (Stetson 1987). Two nearby comparison stars were used and an aperture radius of 6.6" was chosen as it returned the minimum RMS scatter in the out of transit data for WASP-113 b. Initial photometric error estimates were calculated

1 PyRAF is a product of the Space Telescope Science Institute, which is operated by AURA for NASA.

2 IRAF is distributed by the National Optical Astronomy Observatories, which are operated by the Association of Universities for Research in Astronomy, Inc., under cooperative agreement with the National Science Foundation. 
Table 1. Radial velocities of WASP-113 taken with SOPHIE at the OHP.

\begin{tabular}{cccr}
\hline \hline $\begin{array}{c}\text { BJD } \\
-2450000\end{array}$ & $\begin{array}{c}\text { RV } \\
\left(\mathrm{km} \mathrm{s}^{-1}\right)\end{array}$ & $\begin{array}{c} \pm 1 \sigma \\
\left(\mathrm{km} \mathrm{s}^{-1}\right)\end{array}$ & $\begin{array}{r}V_{\text {span }} \\
\left(\mathrm{km} \mathrm{s}^{-1}\right)\end{array}$ \\
\hline 6403.5470 & -11.274 & 0.014 & 0.002 \\
6472.3722 & -11.262 & 0.014 & -0.017 \\
6473.4044 & -11.196 & 0.015 & -0.020 \\
6475.3698 & -11.289 & 0.011 & 0.012 \\
6476.3875 & -11.274 & 0.012 & 0.007 \\
6477.4172 & -11.240 & 0.012 & -0.001 \\
6478.3753 & -11.194 & 0.011 & 0.031 \\
6480.3792 & -11.282 & 0.011 & 0.022 \\
6481.4353 & -11.245 & 0.014 & 0.002 \\
6482.4367 & -11.199 & 0.013 & 0.022 \\
6483.3953 & -11.191 & 0.011 & 0.038 \\
6484.3704 & -11.280 & 0.010 & -0.051 \\
6485.3914 & -11.285 & 0.010 & -0.010 \\
6511.3553 & -11.237 & 0.023 & -0.047 \\
6514.3386 & -11.200 & 0.019 & -0.067 \\
6515.3484 & -11.179 & 0.012 & -0.011 \\
6516.3324 & -11.250 & 0.013 & -0.014 \\
6550.3181 & -11.236 & 0.029 & 0.001 \\
6551.3124 & -11.203 & 0.016 & -0.046 \\
6552.3139 & -11.267 & 0.017 & 0.005 \\
\hline
\end{tabular}

using the electron noise from the target and the sky and the read noise within the aperture.

A transit was partially observed on 2014 June 6 at Oversky Observatory $^{3}$. The telescope is a semi-robotic $0.355 \mathrm{~m}(f / 10)$ Celestron 14-inch Schmidt-Cassegrain installed at Nerpio $(1650 \mathrm{~m})$, Spain. The sequence was interrupted by clouds. The telescope is mounted with Sbig Stl-1001e camera with AOL tip tilt system, containing $1024 \times 1024$ pixels $(24 \mu \mathrm{m}$ in size $)$. The telescope has a field of view of $20 \times 20$ arcmin squared and a pixel scale of $1.18 \sim \operatorname{arcsec} /$ pixel. Its peak quantum efficiency is larger than $72 \%$ at $775 \mathrm{~nm}$. The telescope was not defocused during the acquisitions, and the exposure time was $45 \mathrm{~s}$ with $5 \mathrm{~s}$ dead time between each. Observations were obtained with a Sloan $r^{\prime}$ photometric filter. The data were dark subtracted and flat-field corrected (sky flats) using the Muniwin 2.0 software. Two nearby comparison stars and one check star were used to perform aperture phototmetry and obtain the final light curve.

Two partial transits were observed at Lowell Observatory. The first transit was obtained on 2015 June 6 with the $31^{\prime \prime}$ telescope. The observations had a 60 -s exposure time in the $V$ filter. The night was not photometric and the observations ended prematurely owing to clouds. The second transit was obtained on 2015 July 2016 with the $42^{\prime \prime}$ telescope. The observations had an exposure time of $7 \mathrm{~s}$ in the $V$ filter. Both transits were reduced with the commercial photometry package Canopus ${ }^{4}$. This package includes a photometric catalogue with BVRI data derived from 2MASS JHKs photometry (Warner 2007), as well as more traditional Sloan griz and BVRI photometry catalogues. These data provide photometric zero points and colour indices ( $\sim 0.03 \mathrm{mag})$ for the entire sky via on-chip differential photometry without the need to observe primary standards. Canopus returns absolute magnitudes calculated through a catalogue for all stars in the image.

\footnotetext{
3 http://www. over-sky.fr

4 http://Www .minorplanetobserver.com/MPOSoftware/ MPOSoftware.htm
}

Table 2. Radial velocities of WASP-114 with SOPHIE and CORALIE.

\begin{tabular}{cccr}
\hline \hline $\begin{array}{c}\text { BJD } \\
-2450000\end{array}$ & $\begin{array}{c}\mathrm{RV} \\
\left(\mathrm{km} \mathrm{s}^{-1}\right)\end{array}$ & $\begin{array}{c} \pm 1 \sigma \\
\left(\mathrm{km} \mathrm{s}^{-1}\right)\end{array}$ & $\begin{array}{r}V_{\text {span }} \\
\left(\mathrm{km} \mathrm{s}^{-1}\right)\end{array}$ \\
\hline SOPHIE OHP & & \\
\hline 6504.6181 & -2.372 & 0.014 & 0.024 \\
6505.6035 & -2.866 & 0.011 & -0.015 \\
6507.6168 & -2.424 & 0.013 & 0.003 \\
6508.5416 & -2.862 & 0.013 & -0.048 \\
6509.4731 & -2.354 & 0.020 & -0.150 \\
6510.6115 & -2.519 & 0.014 & -0.045 \\
6513.6136 & -2.624 & 0.017 & -0.099 \\
6514.5991 & -2.792 & 0.021 & -0.024 \\
6515.6156 & -2.378 & 0.013 & -0.023 \\
6516.6464 & -2.730 & 0.024 & -0.145 \\
6533.4146 & -2.873 & 0.011 & -0.008 \\
6534.4568 & -2.523 & 0.012 & -0.043 \\
6535.3847 & -2.557 & 0.012 & -0.037 \\
6536.5472 & -2.861 & 0.012 & -0.029 \\
6538.5287 & -2.496 & 0.012 & -0.035 \\
6550.3639 & -2.831 & 0.029 & - \\
6552.3575 & -2.577 & 0.035 & - \\
6553.4382 & -2.810 & 0.021 & -0.018 \\
\hline \multicolumn{4}{c}{ CORALIE Euler } \\
66486.820396 & -3.201 & 0.032 & 0.034 \\
6490.770491 & -2.628 & 0.032 & -0.013 \\
6505.713259 & -3.037 & 0.027 & -0.026 \\
6508.752985 & -3.141 & 0.029 & -0.020 \\
6511.718158 & -3.181 & 0.029 & -0.032 \\
6516.657808 & -2.988 & 0.035 & 0.101 \\
6517.754717 & -3.127 & 0.039 & 0.148 \\
6531.681814 & -3.048 & 0.054 & -0.195 \\
6532.612125 & -2.656 & 0.055 & -0.018 \\
6533.586138 & -3.061 & 0.045 & -0.040 \\
6538.604777 & -2.705 & 0.048 & -0.102 \\
6540.645293 & -2.807 & 0.089 & -0.167 \\
6544.625775 & -2.888 & 0.033 & -0.080 \\
6545.647037 & -3.142 & 0.025 & -0.011 \\
6558.604624 & -2.841 & 0.028 & -0.037 \\
6561.592328 & -2.953 & 0.037 & 0.034 \\
6571.574752 & -2.765 & 0.029 & 0.099 \\
\hline
\end{tabular}

Another partial transit was obtained with RISE-2 mounted on the $2.3 \mathrm{~m}$ telescope situated at Helmos observatory in Greece on 2015 July 20 . The CCD size is $1 \mathrm{k} \times 1 \mathrm{k}$ pixels with pixel scale of $0.51^{\prime \prime}$ per pixel and a field of view of $9^{\prime} \times 9^{\prime}$ (Boumis et al. 2010). The exposure time was $5 \mathrm{~s}$ and the $V+R$ filter was used. The images were processed with IRAF for bias subtraction and flat fielding. The IRAF DAOPHOT package was used to perform aperture photometry of WASP-113 and the seven comparison stars.

Finally a full transit was obtained with RISE at the Liverpool Telescope on 2016 May 1. The Liverpool telescope is a $2.0 \mathrm{~m}$ robotic telescope located at the Observatorio del Roque de los Muchachos on La Palma. RISE is a back-illuminated, frame transfer CCD which is $1024 \times 1024$ pixels. It uses a " $V+R$ " filter and $2 \times 2$ binning of the detector for all observations. The resulting pixel scale is $1.08 \mathrm{arcsec} / \mathrm{pixel}$. See Steele et al. (2008). We used 2-s exposures and defocused the telescope by $1.0 \mathrm{~mm}$ resulting in a target FWHM of 11.7 pix. Images are automatically bias, dark, and flat corrected by the RISE pipeline. We reduced the data with standard IRAF apphot routines using a 10 pixel (10.8 arcsec) aperture. 
S. C. C. Barros et al.: WASP-113b and WASP-114b, two inflated hot Jupiters with contrasting densities

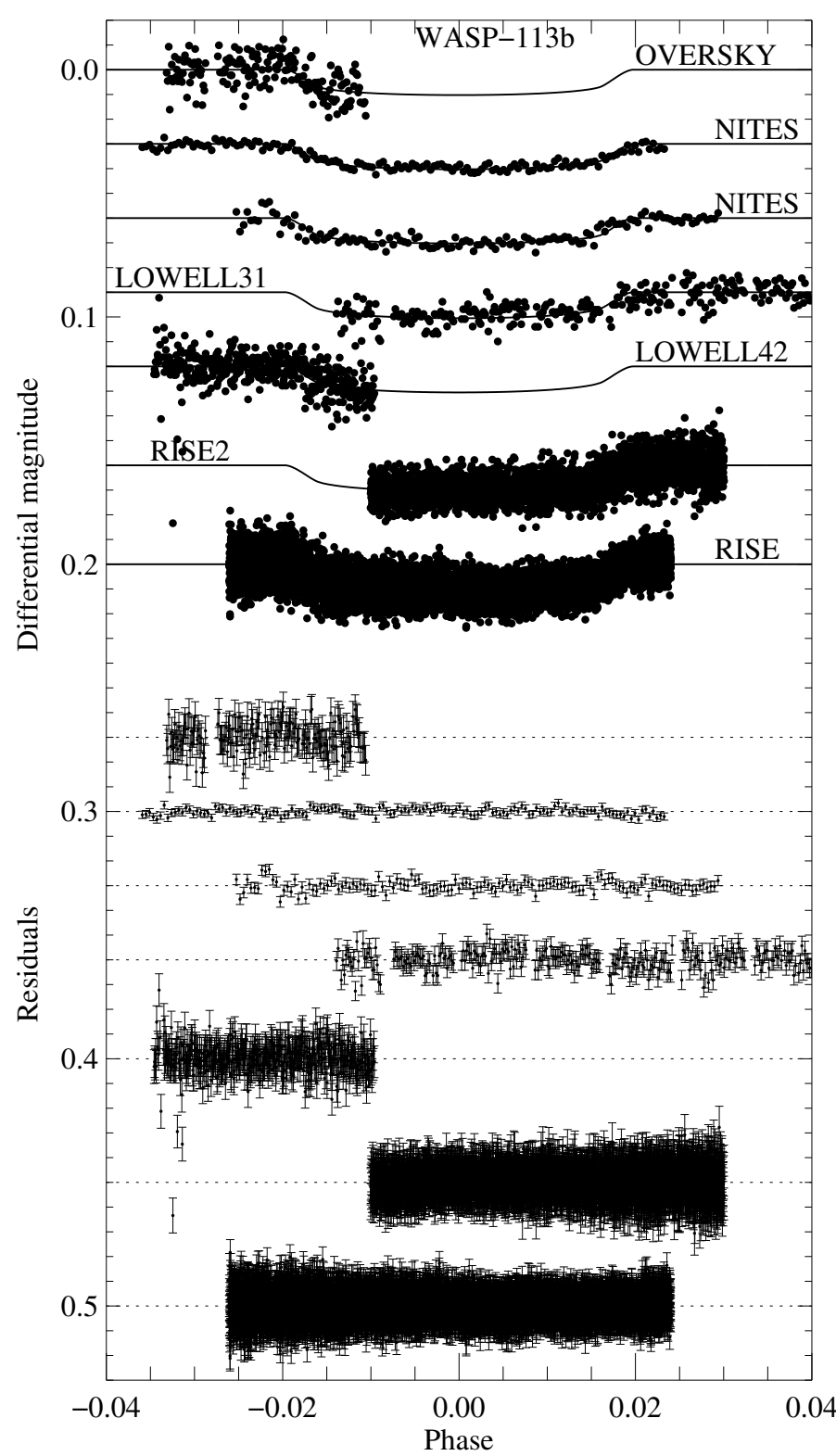

Fig. 4. Phase folded light curves of WASP-113b. From top to bottom: Oversky taken on 2014 June 6, NITES taken on 2014 June 6 and on 2015 March 10, Lowell 31" taken on 2015 July 6, Lowell 42" taken on 2015 July 16, RISE-2 taken on 2015 July 20, and RISE taken on 2016 May 1. For each transit we superimpose the best-fit transit model. The residuals for each light curve are shown in the bottom of the figure. The data were displaced vertically for clarity.

The seven transit light curves of WASP-113 are shown in Fig. 4. We overplot the best-fit model described in detail in Sect. 4.

\subsubsection{WASP-114}

For WASP-114 six transits were obtained. Two transits were obtained with the EulerCam, three transits were obtained with TRAPPIST, and one with RISE-2.

We observed one partial and one full transit of WASP-114 using EulerCam (Lendl et al. 2012) at the $1.2 \mathrm{~m}$ Euler-Swiss telescope located at La Silla observatory. The observations were carried out on 15 September 2013 and 06 July 2014, using an $I$-Cousins filter and exposure times of 60 and 90 s, respectively.
Table 3. Observing log for follow-up transit photometry.

\begin{tabular}{lcc}
\hline \hline Date & Telescope & Filter \\
\hline WASP-113 & & \\
\hline 2014 June 06 & Oversky & $R$ \\
2014 June 06 & NITES & clear \\
2015 March 10 & NITES & clear \\
2015 July 06 & Lowell 31" & $V$ \\
2015 July 16 & Lowell 42 & $V$ \\
2015 July 20 & RISE-2 & $V+R$ \\
2016 May 01 & RISE & $V+R$ \\
\hline WASP-114 & & \\
\hline 2013 September 15 & Euler & $I$ \\
2013 September 29 & TRAPPIST & $I+z$ \\
2014 June 19 & TRAPPIST & $I+z$ \\
2014 July 6 & Euler & $I$ \\
2014 July 20 & TRAPPIST & $I+z$ \\
2014 August 11 & RISE2 & $R$ \\
\hline
\end{tabular}

Conditions were clear during both observations with stellar FWHMs between 1.3 and 2.4 arcsec (15 September 2013) and 1.0 and 1.5 arcsec (06 July 2014). Fluxes were extracted using a photometric aperture of 6.9 arcsec radius, and relative light curves were created using carefully selected reference stars. The final light curves have a residual RMS per 5 min bin of 580 and 490 ppm, respectively.

Three transits of WASP-114b were observed with the $0.6 \mathrm{~m}$ TRAnsiting Planets and PlanetesImals Small Telescope (TRAPPIST robotic telescope) located at ESO La Silla Observatory (Chile). The transits were observed on 29 September 2013, on 19 June 2014, and on 20 July 2014. TRAPPIST is equipped with a thermoelectrically cooled $2 \mathrm{k} \times 2 \mathrm{k} C \mathrm{CD}$, which has a pixel scale of 0.65 that translates into a $22 \times 22$ field of view. For details of TRAPPIST, see Gillon et al. (2011) and Jehin et al. (2011). The transits were observed in a blue-blocking filter ${ }^{5}$ that has a transmittance $>90 \%$ from $500 \mathrm{~nm}$ to beyond $1000 \mathrm{~nm}$. During the runs, the positions of the stars on the chip were maintained to within a few pixels thanks to a software guiding system that regularly derives an astrometric solution for the most recently acquired image and sends pointing corrections to the mount if needed. After a standard pre-reduction (bias, dark, and flatfield correction), the stellar fluxes were extracted from the images using the IRAF/DAOPHOT2 aperture photometry software (Stetson 1987). For each light curve, we tested several sets of reduction parameters and kept the one giving the most precise photometry for the stars of similar brightness to the target. After a careful selection of reference stars, the transit light curves were finally obtained using differential photometry.

One more transit of WASP114b was observed with RISE2 mounted on the $2.3 \mathrm{~m}$ telescope situated at Helmos observatory in Greece and already described above. The images were processed with IRAF for bias subtraction and flat fielding. The IRAF DAOPHOT package was used to perform aperture photometry of WASP-114 and the six comparison stars. There is evidence for systematics in the light curve as was also seen in the light curves taken with RISE at the Liverpool Telescope (Barros et al. 2011b). We performed the fit with and without this light curve and confirmed that the light curve does not bias the final results.

In Fig. 5 we show the six high quality transit light curves of WASP-114. The best-fit model is also overplotted.

http://www . astrodon.com/products/filters/exoplanet/ 


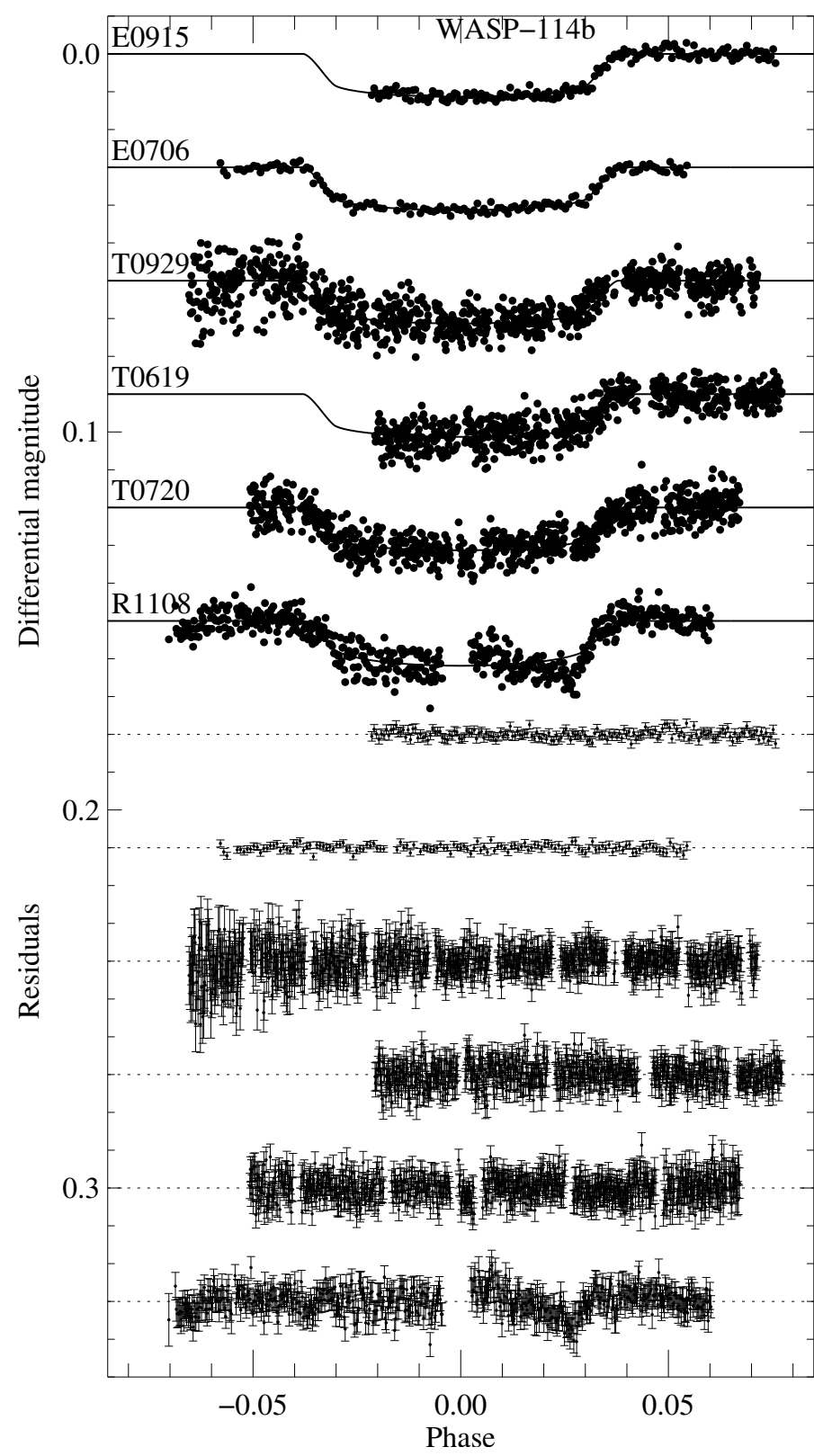

Fig. 5. Phase folded light curve of WASP-114b. From top to bottom: EulerCam observations taken on 2013 September 15 and 2014 July 6; TRAPPIST observations taken on 2013 September 29, 2014 June 19 , and 2014 July 20; and RISE-2 observation taken on 2014 August 11. The best-fit transit model is superimposed on the transits and the residuals of the best-fit model for each light curve are shown in the bottom of the figure. The data were displaced vertically for clarity

\section{Spectral characterisation of the host stars}

The individual SOPHIE spectra were radial velocity corrected and co-added, giving average $\mathrm{S} / \mathrm{N}$ ratios of $\sim 80: 1$ for both stars. The spectral analysis was performed using the procedures detailed in Doyle et al. (2013).

For each star the effective temperature $\left(T_{\text {eff }}\right)$ was obtained using the $\mathrm{H} \alpha$ line and surface gravity $(\log g)$ determined from the $\mathrm{Na} \mathrm{D}$ and $\mathrm{Mg} \mathrm{b}$ lines. Iron abundances were obtained from the analysis of equivalent width measurements of several unblended Fe I lines. Projected rotation velocities $(v \sin I)$ were determined by fitting the profiles of the $\mathrm{Fe} I$ lines after convolving with the instrumental resolution $(R=75000)$ and a macroturbulent velocity adopted from the calibration of
Table 4. Stellar parameters of WASP-113 and WASP114 from spectroscopic analysis.

\begin{tabular}{lll}
\hline \hline Parameter & WASP-113 & WASP-114 \\
\hline RA(J2000) & $14: 59: 29.49$ & $21: 50: 39.74$ \\
Dec(J2000) & $+46: 57: 36.4$ & $+10: 27: 46.9$ \\
$V[\mathrm{mag}]$ & $11.771 \pm 0.045$ & $12.743 \pm 0.148$ \\
$T_{\text {eff }}[\mathrm{K}]$ & $5890 \pm 140$ & $5940 \pm 140$ \\
$\log g[\mathrm{cgs}]$ & $4.2 \pm 0.1$ & $4.3 \pm 0.1$ \\
{$[\mathrm{Fe} / \mathrm{H}]$} & $0.10 \pm 0.09$ & $0.14 \pm 0.07$ \\
$v \sin I\left[\mathrm{~km} \mathrm{~s}^{-1}\right]$ & $6.8 \pm 0.7$ & $6.4 \pm 0.7$ \\
$\log \mathrm{A}(\mathrm{Li})$ & $2.03 \pm 0.12$ & $1.77 \pm 0.12$ \\
Spectral type & $\mathrm{G} 1$ & $\mathrm{G} 0$ \\
Distance $[\mathrm{pc}]$ & $360 \pm 70$ & $460 \pm 80$ \\
\hline
\end{tabular}

Notes. Spectral type estimated from $T_{\text {eff }}$ and Table B1 of Gray (2008).

Doyle et al. (2014). We also estimated $v \sin I$ from the CCF using the procedure of Boisse et al. (2010) and found consistent results: $5.8 \pm 0.1 \mathrm{~km} \mathrm{~s}^{-1}$ and $4.7 \pm 0.1 \mathrm{~km} \mathrm{~s}^{-1}$ for WASP-113 and WASP-114, respectively.

Furthermore, we estimated the spectral type using Teff and Table B1 of Gray (2008). The derived stellar parameters for WASP-113 and WASP-114 are given in Table 4. We find that the stellar parameters of WASP-113 and WASP-114 are very similar.

\subsection{Stellar age}

Combining the $v \sin i$ with the stellar radius we derive an upper limit to the stellar rotation period of $10.56 \pm 1.8$ days and $9.96 \pm$ 1.73 days for WASP-113 and WASP-114, respectively. These allow us to derive an upper limit to the gyrochronological age of $1.04_{-0.23}^{+0.49}$ Gyr for WASP-113 and $0.98_{-0.31}^{+0.51}$ Gyr for WASP-114 using Barnes \& Kim (2010). However, the lithium abundance suggests an age of $\sim 5 \mathrm{Gyr}$ for both stars (Sestito \& Randich 2005). A discrepancy between the age indicators is not unusual (Brown 2014; Maxted et al. 2015); however, the reason is still not clear.

A more precise constraint on the age can be obtained by using theoretical stellar models. We follow the procedure of Brown (2014) using three sets of stellar models: the Padova models of Marigo et al. (2008) and Bressan et al. (2012), the Yonsei-Yale (YY) isochrones of Demarque et al. (2004), and the Dartmouth Stellar Evolution Database (DSED) models of Dotter et al. (2008). We use the values of $T_{\text {eff }}$ and $[\mathrm{Fe} / \mathrm{H}] \mathrm{de}-$ rived from the spectroscopic analyses and the transit derived stellar density whose derivation is explained in the next section. For WASP-113 we found an age of $6.4_{-10.9}^{+2} \mathrm{Gyr}, 5.1_{-1.7}^{+3.3} \mathrm{Gyr}$, and $7.2_{-3.1}^{+2.9}$ Gyr respectively for the models of Padova, YY, and DSED. In the left panel of Fig. 6 we show the isochrones and mass tracks for WASP-113 for the YY models. According to these models WASP-113 has a mass of $1.19_{-0.13}^{+0.10} M_{\odot}$, which is very close to the critical mass for the stellar cores to become convective. Owing to the convective cores, the evolution and consequently the shape of the evolutionary tracks for higher mass stars is significantly different from lower mass stars, as can be seen in this figure. In this region of the parameter space details on the treatment of convective overshooting become important and the uncertainty in the stellar age and mass are higher. We find that WASP-113 is at the end of its main sequence life and could be already in the hydrogen-shell burning phase. For WASP-114 we obtained an age of $3.9_{-2.3}^{+2.4} \mathrm{Gyr}, 4.2_{-1.4}^{+1.7} \mathrm{Gyr}$, and $4.9_{-2.7}^{+3.1} \mathrm{Gyr}$ respectively for the models of Padova, YY, and DSED, which 

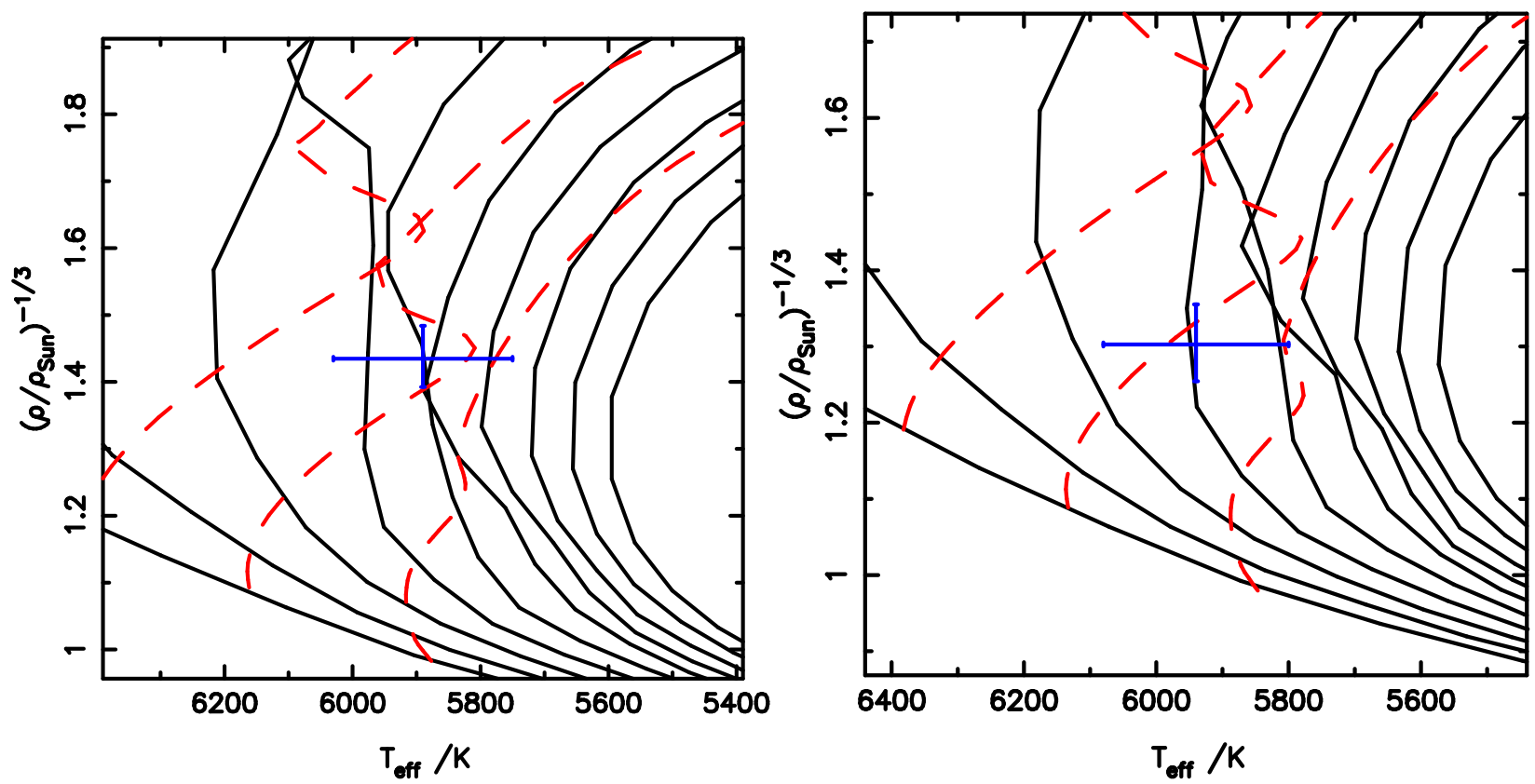

Fig. 6. Modified H-R diagrams for WASP-113 on the left and WASP-114 on the right. We display the stellar models of YY (Demarque et al. 2004). The isochrones are shown solid black line (for 1,2,3,4,5,6, 7, 8,9,10 Gyr old stars from left to right) and and the mass tracks in red (from left to right we show $1.3,1.2$, and $1.1 M_{\odot}$ ) for the corresponding metallicity given in Table 4 .

agrees with the age estimate from the lithium abundance. The isochrones and mass tracks for WASP-114 are shown in the right panel of Fig. 6. According to the models WASP-114 also has a mass close to the critical limit $1.18 \pm 0.10 M_{\odot}$, but the star is younger than WASP-113. WASP-114 is still in the middle of its main sequence life where the differences between the evolution of lower mass and higher mass stars are smaller.

\section{Derivation of the system parameters}

To derive the system parameters we used an updated version of the Markov chain Monte Carlo (MCMC) fitting procedure described by Collier Cameron et al. (2007) and Pollacco et al. (2008). The photometry and the radial velocity measurements are fitted simultaneously. The transit model is based on the Mandel \& Agol (2002) model parametrised by the transit epoch $T_{0}$, orbital period $P$, impact parameter $b$, transit duration $T_{T}$, and squared ratio of planet radius to star radius $\left(R_{\mathrm{p}} / R_{*}\right)^{2}$. For each photometric data set, we include the non-linear limb darkening coefficients for the respective filter based on the tables of Claret $(2000,2004)$. The host star's reflex motion is modelled with a Keplerian parametrised by the centre-of-mass velocity $\gamma$, the radial velocity amplitude $K$, an offset between SOPHIE and CORALIE, $\sqrt{e} \cos (\omega)$ and $\sqrt{e} \sin (\omega)$, where $e$ is the orbital eccentricity and $\omega$ the longitude of the periastron. The parameters $\sqrt{e} \cos (\omega)$ and $\sqrt{e} \sin (\omega)$ are used in order to impose a uniform prior in the eccentricity.

The Torres et al. (2010) empirical calibration is used to estimate the stellar mass and radius from the spectroscopic derived $T_{\text {eff }}$ and $[\mathrm{Fe} / \mathrm{H}]$, and from the $\rho_{*}$ measured from the transit as described in Enoch et al. (2010). In general the Torres et al. (2010) empirical calibration was found to be in good agreement with stellar models and it has the advantage that it can be directly used in the transit fitting procedure. For our case we also find a good agreement between the stellar mass derived using the Torres et al. (2010) empirical calibration and that estimated using the stellar models.
The system parameters for WASP-113 and WASP-114 and the $1 \sigma$ uncertainties derived from the MCMC analysis are given in Table 5. For both WASP-113 and WASP-114 the transits allow a good constraint on the stellar density. Moreover, the transit derived $\log g$ (for WASP113 $\log g=4.14 \pm 0.05$, for WASP114 $\log g=4.24 \pm 0.03$ ) agrees well with the spectroscopic measured $\log g$ (Table 4). We find that although both stars have a similar mass, WASP-113 has a lower density and larger radius confirming that WASP-113 is more evolved than WASP-114.

We find a hint of eccentricity for WASP-113 of $0.16_{-0.10}^{+0.12}$, not significant at $2 \sigma$. The Lucy and Sweeney test (Lucy \& Sweeney 1971) gives a high probability of the eccentricity occurring by chance. Since this value of the eccentricity consistent with zero is actually non-zero it affects the transit derived stellar density changing it by slightly more than $1 \sigma$ and biasing the system parameters. Therefore, we force a circular orbit for WASP-113b.

For WASP-114, the eccentricity was found to be $0.01_{-0.01}^{+0.02}$, hence a circular orbit is favoured. However, in this case the eccentricity is much better constrained and the system parameters are not biased. The values of the parameters agree within $0.1 \sigma$ for the circular and eccentric case and hence we adopt the eccentric model to obtain more reliable parameter uncertainties which are slightly bigger when the eccentricity is left free.

We conclude that WASP-113b is a $0.475 M_{\text {Jup }}$ planet with a $1.409 R_{\text {Jup }}$ radius and a low density $0.172 \rho_{\mathrm{J}}$ in a 4.54217 day orbit and WASP-114b is a $1.769 M_{\text {Jup }}$ giant planet with a $1.339 R_{\text {Jup }}$ radius and a density of $0.73 \rho_{\mathrm{J}}$ in a 1.54877 -day orbit.

\section{Discussion}

We present the discovery and characterisation of WASP-113b, a hot Jupiter in a 4.5-day orbit around a G1-type star, and of WASP-114b, a hot Jupiter in a 1.5-day orbit around a G0 star. WASP-113, with $T_{\text {eff }}=5890 \mathrm{~K}, \log g=4.2$, and $[\mathrm{Fe} / \mathrm{H}]=0.10$, is orbited by a $0.475 M_{\text {Jup }}$ planet with a radius of $1.409 R_{\text {Jup }}$ and hence a density of $0.172 \rho_{\mathrm{J}}$. WASP-114 has a $T_{\text {eff }}=5940 \mathrm{~K}$, $\log g=4.3$, and $[\mathrm{Fe} / \mathrm{H}]=0.14$, and the planetary companion 
Table 5. WASP-113 and WASP-114 system parameters.

\begin{tabular}{|c|c|c|}
\hline Parameter & WASP-113 & WASP-114 \\
\hline Transit epoch $T_{0}[\mathrm{HJD}]$ & $2457197.097459 \pm 0.000040$ & $2456667.73582 \pm 0.00021$ \\
\hline Orbital period $P$ [days] & $4.54216874538 \pm 0.0000042$ & $1.5487743_{-0.00000091}^{+0.0000012}$ \\
\hline Planet/star area ratio $\left(R_{\mathrm{p}} / R_{*}\right)^{2}$ & $0.00809 \pm 0.00028$ & $0.00927 \pm 0.00016$ \\
\hline Transit duration $T_{\mathrm{T}}$ [days] & $0.1791 \pm 0.0019$ & $0.11592 \pm 0.00079$ \\
\hline Impact parameter $b\left[R_{*}\right]$ & $0.486_{-0.14}^{+0.063}$ & $0.457_{-0.054}^{+0.052}$ \\
\hline Orbital inclination $I$ [deg] & $86.46_{-0.64}^{+1.2}$ & $83.96 \pm 0.90$ \\
\hline Stellar reflex velocity $K\left[\mathrm{~m} \mathrm{~s}^{-1}\right]$ & $48.74 \pm 5.2$ & $260.6 \pm 5.4$ \\
\hline Orbital semi-major axis $a$ [au] & $0.05885 \pm 0.00010$ & $0.02851 \pm 0.00039$ \\
\hline Orbital eccentricity $e$ & fixed $=0$ & $0.012_{-0.009}^{+0.022}$ \\
\hline Longitude of periastron $\omega\left[^{\circ}\right]$ & fixed $=0$ & $-71 \cdot_{-35}^{+150}$ \\
\hline $\begin{array}{l}\text { Stellar mass } M_{*}\left[M_{\odot}\right] \\
\text { Stellar radius } R_{*}\left[R_{\odot}\right]\end{array}$ & $\begin{array}{c}1.318 \pm 0.069 \\
1.608_{-0.12}^{+0.090}\end{array}$ & $\begin{array}{c}1.289 \pm 0.053 \\
1.43 \pm 0.060\end{array}$ \\
\hline Stellar density $\rho_{*}\left[\rho_{\odot}\right]$ & $0.317_{-0.039}^{0.072}$ & $0.439_{-0.041}^{+0.050}$ \\
\hline Planet mass $M_{\mathrm{p}}\left[M_{\mathrm{Jup}}\right]$ & $0.475_{-0.052}^{+0.054}$ & $1.769 \pm 0.064$ \\
\hline Planet radius $R_{\mathrm{p}}\left[R_{\mathrm{Jup}}\right]$ & $1.409_{-0.14}^{+0.096}$ & $1.339 \pm 0.064$ \\
\hline Planet density $\rho_{\mathrm{p}}\left[\rho_{\mathrm{J}}\right]$ & $0.172_{-0.034}^{+0.055}$ & $0.73 \pm 0.10$ \\
\hline Planet surface gravity $\log g_{\mathrm{p}}[\mathrm{cgs}]$ & $2.744_{-0.072}^{+0.081}$ & $3.353 \pm 0.036$ \\
\hline Planet temperature $T_{\text {eq }}[\mathrm{K}]$ & $1496 \pm 60$ & $2043 \pm 58$ \\
\hline
\end{tabular}

is more massive with a mass of $1.769 M_{\mathrm{Jup}}$ and a radius of $1.339 R_{\mathrm{Jup}}$ and hence a density of $0.73 \rho_{\mathrm{J}}$.

A circular orbit is preferred for both planets, which is not surprising given that the circularisation timescale (Goldreich \& Soter 1966; Bodenheimer et al. 2001) is much shortter than the age of the host stars. Using Eq. (2) in Bodenheimer et al. (2001) and assuming $Q_{\mathrm{p}}=10^{5}-10^{6}$ (Levrard et al. 2009), we compute the circularisation timescale for WASP-113b, $\tau_{\text {cir }}=0.016-0.16 \mathrm{Gyr}$, and for WASP-114b, $\tau_{\text {cir }}=0.00073-0.0073$ Gyr. These are much shorter than the host stars ages derived from spectra $(\sim 6.2 \mathrm{Gyr}$ and $\sim 4.3 \mathrm{Gyr}$ for WASP-113 and WASP-114, respectively), thus favouring circular orbits.

The mass-radius relationship for giant planets depends on their internal composition since heavier elements decrease the planetary radius. Assuming the coreless models of Fortney et al. (2007) we estimated a radius of $1.05 R_{\text {Jup }}$ for WASP-113b and $1.15 R_{\mathrm{Jup}}$ for WASP-114b. Both these predicted radii are more than $2 \sigma$ smaller than the radii measured for the planets in our analysis. Hence, we conclude that the planets are inflated. Following Laughlin et al. (2011) we compute a radius anomaly, $\mathfrak{R}=0.35$, for WASP $-113 b$ and $\mathfrak{R}=0.189$ for WASP-114b. Recently it has become clear that regardless of the nature of the inflation mechanism, there is a clear correlation between the stellar incident flux and the radius anomaly (Laughlin et al. 2011; Weiss et al. 2013). Furthermore, giant planets that receive modest stellar flux do not show a radius anomaly (Demory \& Seager 2011). At first glance the radius anomaly of WASP-113b and WASP-114b seem to contradict this correlation since the effective temperature of WASP$114 \mathrm{~b}$ is higher than WASP-113b. In fact, WASP-113b has a radius anomaly above the mean relationship proposed by Laughlin et al. (2011; $\left.\mathfrak{R} \propto T_{\text {equ }}^{1.4}\right)$, while WASP-114b has a radius anomaly slightly lower than this mean scaling relationship. However, other exoplanets share the same properties as WASP$113 \mathrm{~b}$ and WASP-114b, and the differences of radius anomaly can be explained by the planetary mass. WASP-114 is 3.7 times more massive than WASP-113. Therefore, it probably has a much higher amount of planetary heavy elements and its higher gravitational binding energy counteracts the inflation. The known exoplanets in the mass range of $0.5 M_{\odot}$ and $1.5 M_{\odot}$ have the largest known radii (Lopez \& Fortney 2016) in agreement with the higher radius anomaly of WASP-113b.

There are several theories to explain the inflated radii in hot Jupiters: tidal heating (Bodenheimer et al. 2001), enhanced atmospheric opacities (Burrows et al. 2007), kinetic heating due to winds (Guillot \& Showman 2002), and ohmic dissipation (Batygin et al. 2011). The last two are mechanisms related to incident stellar flux and are favoured by the recent reported correlation. However, there is no theory capable of explaining all the measured radius anomalies (e.g. Spiegel \& Burrows 2013). Owing to the old age of both WASP-113 and WASP-114 compared 
with the circularisation timescale, tidal heating would not be expected to play an important role, hence a combination of the other mechanisms is more likely.

WASP-113 is at the end of its main sequence life. We estimate that owing the expansion of the star after the end of the main sequence the planet will be engulfed in $\sim 1.4 \mathrm{Gyr}$. The response of the planetary radius to the increase in the stellar radius and stellar luminosity will distinguish mechanisms that are directly capable of inflating the planet from those that only slow down the cooling exoplanets (Lopez \& Fortney 2016). An alternative to this long wait is to search for hot Jupiters with periods of 10-30 days around evolved stars (Lopez \& Fortney 2016).

Follow-up observations of these planets can help shed light on the radius anomaly and on the atmospheric composition of these planets. The large scale-height of WASP-113b, $\sim 950 \mathrm{~km}$, and its relatively bright host star, $V=11.8$, makes it a good target for transmission spectroscopy observations to probe its atmospheric composition. The scale height of WASP-114b is smaller $(\sim 310 \mathrm{~km})$ and combined with its fainter host star would make atmospheric studies more challenging.

Acknowledgements. The WASP Consortium consists of astronomers primarily from Queen's University Belfast, St Andrews, Keele, Leicester, The Open University, Isaac Newton Group La Palma, and Instituto de Astrofsica de Canarias. The SuperWASP-N camera is hosted by the Issac Newton Group on La Palma. We are grateful for their support and assistance. Funding for WASP comes from consortium universities and from the UK's Science and Technology Facilities Council. Based on observations made at Observatoire de Haute Provence (CNRS), France, and at the ESO La Silla Observatory (Chile) with the CORALIE Echelle spectrograph mounted on the Swiss telescope. We thank the staff at Haute-Provence Observatory. S.C.C.B. acknowledges support by grant 98761 from CNES and the Fundação para a Ciência e a Tecnologia (FCT) through the Investigador FCT Contract No. IF/01312/2014 and the grant reference PTDC/FIS-AST/1526/2014. D.J.A., D.P., and C.W. acknowledge funding from the European Union Seventh Framework programme (FP7/2007-2013) under grant agreement No. 313014 (ETAEARTH). The Swiss Euler Telescope is operated by the University of Geneva, and is funded by the Swiss National Science Foundation. The Aristarchos telescope is operated on Helmos Observatory by the Institute for Astronomy, Astrophysics, Space Applications and Remote Sensing of the National Observatory of Athens. TRAPPIST is a project funded by the Belgian Fund for Scientific Research (Fond National de la Recherche Scientifique, F.R.S-FNRS) under grant FRFC 2.5.594.09.F, with the participation of the Swiss National Science Fundation (SNF). M. Gillon and E. Jehin are FNRS Research Associates. L. Delrez acknowledges support of the F.R.I.A. fund of the FNRS.

\section{References}

Baranne, A., Queloz, D., Mayor, M., et al. 1996, A\&AS, 119, 373

Barnes, S. A., \& Kim, Y.-C. 2010, ApJ, 721, 675

Barros, S. C. C., Faedi, F., Collier Cameron, A., et al. 2011a, A\&A, 525, A54

Barros, S. C. C., Pollacco, D. L., Gibson, N. P., et al. 2011b, MNRAS, 416, 2593

Barros, S. C. C., Demangeon, O., \& Deleuil, M. 2016, A\&A, in press, DOI: $10.1051 / 0004-6361 / 201628902$

Batygin, K., Stevenson, D. J., \& Bodenheimer, P. H. 2011, ApJ, 738, 1 Bodenheimer, P., Lin, D. N. C., \& Mardling, R. A. 2001, ApJ, 548, 466 Boisse, I., Eggenberger, A., Santos, N. C., et al. 2010, A\&A, 523, A88

Borucki, W. J., Koch, D., Basri, G., et al. 2010, Science, 327, 977
Bouchy, F., Hébrard, G., Udry, S., et al. 2009, A\&A, 505, 853

Boumis, P., Pollacco, D., Steele, I., et al. 2010, in 9th International Conference of the Hellenic Astronomical Society, eds. K. Tsinganos, D. Hatzidimitriou, \& T. Matsakos, ASP Conf. Ser., 424, 426

Bressan, A., Marigo, P., Girardi, L., et al. 2012, MNRAS, 427, 127

Brown, D. J. A. 2014, MNRAS, 442, 1844

Burrows, A., Hubeny, I., Budaj, J., \& Hubbard, W. B. 2007, ApJ, 661, 502

Charbonneau, D., Brown, T. M., Latham, D. W., \& Mayor, M. 2000, ApJ, 529, L45

Charbonneau, D., Brown, T. M., Noyes, R. W., \& Gilliland, R. L. 2002, ApJ, 568,377

Charbonneau, D., Knutson, H. A., Barman, T., et al. 2008, ApJ, 686, 1341

Claret, A. 2000, A\&A, 363, 1081

Claret, A. 2004, A\&A, 428, 1001

Collier Cameron, A., Pollacco, D., Street, R. A., et al. 2006, MNRAS, 373, 799

Collier Cameron, A., Wilson, D. M., West, R. G., et al. 2007, MNRAS, 380, 1230

Demarque, P., Woo, J., Kim, Y., \& Yi, S. K. 2004, ApJS, 155, 667

Deming, D., Seager, S., Richardson, L. J., \& Harrington, J. 2005, Nature, 434, 740

Demory, B.-O., \& Seager, S. 2011, ApJS, 197, 12

Dotter, A., Chaboyer, B., Jevremović, D., et al. 2008, ApJS, 178, 89

Doyle, A. P., Smalley, B., Maxted, P. F. L., et al. 2013, MNRAS, 428, 3164

Doyle, A. P., Davies, G. R., Smalley, B., Chaplin, W. J., \& Elsworth, Y. 2014 MNRAS, 444, 3592

Enoch, B., Collier Cameron, A., Parley, N. R., \& Hebb, L. 2010, A\&A, 516, A33

Fortney, J. J., Marley, M. S., \& Barnes, J. W. 2007, ApJ, 659, 1661

Gillon, M., Jehin, E., Magain, P., et al. 2011, in EPJ Web Conf., 11, 6002

Goldreich, P., \& Soter, S. 1966, Icarus, 5, 375

Gray, D. F. 2008, The Observation and Analysis of Stellar Photospheres (Cambridge, UK: CUP)

Grillmair, C. J., Burrows, A., Charbonneau, D., et al. 2008, Nature, 456, 767

Guillot, T. 2005, Ann. Rev. Earth Planet. Sci., 33, 493

Guillot, T., \& Showman, A. P. 2002, A\&A, 385, 156

Henry, G. W., Marcy, G. W., Butler, R. P., \& Vogt, S. S. 2000, ApJ, 529, L41

Howell, S. B., Sobeck, C., Haas, M., et al. 2014, PASP, 126, 398

Jehin, E., Gillon, M., Queloz, D., et al. 2011, The Messenger, 145, 2

Kovács, G., Bakos, G., \& Noyes, R. W. 2005, MNRAS, 356, 557

Laughlin, G., Crismani, M., \& Adams, F. C. 2011, ApJ, 729, L7

Lendl, M., Anderson, D. R., Collier-Cameron, A., et al. 2012, A\&A, 544, A72

Levrard, B., Winisdoerffer, C., \& Chabrier, G. 2009, ApJ, 692, L9

Lopez, E. D., \& Fortney, J. J. 2016, ApJ, 818, 4

Lucy, L. B., \& Sweeney, M. A. 1971, AJ, 76, 544

Mandel, K., \& Agol, E. 2002, ApJ, 580, L171

Marigo, P., Girardi, L., Bressan, A., et al. 2008, A\&A, 482, 883

Maxted, P. F. L., Serenelli, A. M., \& Southworth, J. 2015, A\&A, 577, A90

McCormac, J., Skillen, I., Pollacco, D., et al. 2014, MNRAS, 438, 3383

Pepe, F., Mayor, M., Galland, F., et al. 2002, A\&A, 388, 632

Perruchot, S., Kohler, D., Bouchy, F., et al. 2008, in SPIE Conf. Ser., 7014

Pollacco, D. L., Skillen, I., Collier Cameron, A., et al. 2006, PASP, 118, 1407

Pollacco, D., Skillen, I., Collier Cameron, A., et al. 2008, MNRAS, 385, 1576

Queloz, D., Mayor, M., Weber, L., et al. 2000, A\&A, 354, 99

Sestito, P., \& Randich, S. 2005, A\&A, 442, 615

Spiegel, D. S., \& Burrows, A. 2013, ApJ, 772, 76

Steele, I. A., Bates, S. D., Gibson, N., et al. 2008, in SPIE Conf. Ser. 7014

Stetson, P. B. 1987, PASP, 99, 191

Tamuz, O., Mazeh, T., \& Zucker, S. 2005, MNRAS, 356, 1466

Torres, G., Andersen, J., \& Giménez, A. 2010, A\&ARv, 18, 67

Vanderburg, A., \& Johnson, J. A. 2014, PASP, 126, 948

Vidal-Madjar, A., Lecavelier des Etangs, A., Désert, J., et al. 2003, Nature, 422, 143

Warner, B. D. 2007, Minor Planet Bulletin, 34, 113

Weiss, L. M., Marcy, G. W., Rowe, J. F., et al. 2013, ApJ, 768, 14 